\title{
Somatostatin receptor expression indicates improved prognosis in gastroenteropancreatic neuroendocrine neoplasm, and octreotide long-acting release is effective and safe in Chinese patients with advanced gastroenteropancreatic neuroendocrine tumors
}

\author{
YUHONG WANG ${ }^{1 *}$, WEI WANG ${ }^{2 *}$, KAIZHOU JIN ${ }^{3}$, CHENG FANG $^{2}$, YUAN LIN $^{4}$, LING XUE $^{4}$,

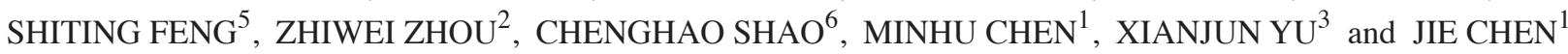 \\ ${ }^{1}$ Department of Gastroenterology, The First Affiliated Hospital, Sun Yat-Sen University; \\ ${ }^{2}$ Department of Gastric and Pancreatic Surgery, Sun Yat-sen University Cancer Center, Guangzhou, \\ Guangdong 510080; ${ }^{3}$ Department of Pancreatic Oncology, Shanghai Cancer Center, Fudan University, \\ Shanghai 200032; Departments of ${ }^{4}$ Pathology and ${ }^{5}$ Radiology, The First Affiliated Hospital, Sun \\ Yat-Sen University, Guangzhou, Guangdong 510080; ${ }^{6}$ Department of General Surgery, Changzheng \\ Hospital, Second Military Medical University, Shanghai 200032, P.R. China
}

Received April 27, 2016; Accepted November 28, 2016

DOI: $10.3892 / \mathrm{ol} .2017 .5591$

\begin{abstract}
Gastroenteropancreatic neuroendocrine neoplasm (GEP-NEN) is known to overexpress somatostatin receptors (SSTRs), most commonly SSTR2 and SSTR5. The expression of SSTRs on tumor cells forms the basis for somatostatin analog treatment of patients with NEN. The present study detected the expression of SSTR2 and SSTR5 in GEP-NEN and investigated the efficacy and safety of octreotide long-acting release
\end{abstract}

Correspondence to: Dr Xianjun Yu, Department of Pancreatic Oncology, Shanghai Cancer Center, Fudan University, 270 Dong An Road, Shanghai 200032, P.R. China

E-mail: yuxianjun@fudanpci.org

Dr Jie Chen, Department of Gastroenterology, The First Affiliated Hospital, Sun Yat-Sen University, 58 Zhongshan II Road, Guangzhou, Guangdong 510080, P.R. China

E-mail: chen0jie@hotmail.com

*Contributed equally

Abbreviations: SSTRs, somatostatin receptors; GEP-NENs, gastroenteropancreatic neuroendocrine neoplasms; IHC, immunohistochemistry; SSA, somatostatin analog; GEP-NET, gastroenteropancreatic neuroendocrine tumor; LAR, long-acting release; TTP, time to progression; GI, gastrointestinal; RECIST, response evaluation criteria in solid tumors; OS, overall survival; ORR, objective response rate; SD, stable disease; CI, confidence interval; NEC, neuroendocrine carcinoma; MANEC, mixed adenoneuroendocrine carcinoma; NR, not reached; $\mathrm{PD}$, progressive disease; PR, partial remission; AEs, adverse events; SAE, serious adverse event

Key words: gastroenteropancreatic neuroendocrine neoplasm, somatostatin receptors, octreotide long-acting release, treatment
(LAR) in the treatment of advanced gastroenteropancreatic neuroendocrine tumors (GEP-NET) in China. The present study reported that functionality of the pancreas, G1 and G2 grading, NET classification and Tumor-Node-Metastasis stages I and II were associated with higher SSTR2 positive expression. Similarly, SSTR5 was increased in pancreatic and well-differentiated tumors. SSTR2 and SSTR5 positive expression predicted improved survival in GEP-NEN patients. The median overall survival of patients treated with octreotide LAR was not reached. The median time to progression was 20.2 months, with the objective response rate being $5.6 \%$ and the stable disease rate being $79.6 \%$. A total of $25.9 \%$ of the patients experienced adverse drug reactions. In conclusion, the present study demonstrated that SSTR2 and SSTR5 are heterogeneously expressed in GEP-NEN. Both markers may serve as potential prognostic factors. Octreotide LAR is effective and safe in the treatment of Chinese patients with advanced GEP-NET.

\section{Introduction}

Gastroenteropancreatic neuroendocrine neoplasms (GEP-NENs) constitute a heterogeneous group of neoplasms, with various clinical presentations and biological behaviors that present diagnostic and therapeutic challenges. As 60-80\% of patients present with metastatic disease at the time of diagnosis, they are treated with multidisciplinary approaches for symptom control and inhibition of tumor growth (1).

Neuroendocrine tumors are known to overexpress somatostatin receptors (SSTRs), a family of G protein-coupled-receptors, most commonly SSTR2 and SSTR5 (2). In previous studies, SSTRs have been extensively mapped in neuroendocrine tumors, using reverse transcription-polymerase chain reaction, autoradiography and immunoblotting (2-4). To date, few studies have examined 
the expression of SSTRs in GEP-NEN by means of immunohistochemistry (IHC), which allows precise SSTR cellular localization $(5,6)$. Furthermore, whether or not differences in the expression of SSTR subtypes in GEP-NEN are associated with tumor characteristics remains to be elucidated, as does the potential prognostic role played by the expression of SSTRs in these tumors.

The expression of SSTRs on tumor cells forms the basis for somatostatin analog (SSA) treatment of patients with NEN (7). SSAs are an important means of biotherapy. They are a class of artificially synthesized peptides that have multiple biological effects similar to natural somatostatin (8). SSA is able to either inhibit the release of hormones and neurotransmitters by binding SSTRs to improve symptoms caused by excessive secretion of hormones (9), or inhibit tumor growth directly by regulating the signaling pathways of tumor cell proliferation/apoptosis and angiogenesis directly or indirectly (10). At present, clinical treatment of gastroenteropancreatic neuroendocrine tumor (GEP-NET) is mainly focused on long-acting SSAs, including octreotide long-acting release (LAR) and lanreotide sustained-release (Somatuline Autogel). The results of a phase III prospective, randomized and double-blind study (PROMID) proved that octreotide LAR significantly prolonged the time to progression (TTP; 14.3 vs. 6 months) in patients with unresectable, well-differentiated metastatic midgut neuroendocrine tumors, as compared with the placebo (11). The results of the CLARINET study, which included 204 patients with non-functional, metastatic NET (including those with a Ki67 $<10 \%$ for tumors in the gastrointestinal tract and the pancreas), showed that the median progression-free survival was not reached in the lanreotide group and was 18.0 months in the placebo group, with the difference between the two groups being statistically significant $(\mathrm{P}<0.001)(12)$.

However, patients in the above-mentioned studies were all from Western countries rather than Asian countries. Previous studies revealed that GEP-NEN is a type of tumor with high heterogeneity; the primary site and symptoms of the tumor vary from patients of various races in differing regions (13-16). In addition, the response and tolerance of GEP-NEN patients to anti-tumor drug treatment also varied between different races $(17,18)$. Therefore, although numerous studies in Western population reported that SSA had anti-tumor activity against GEP-NET, considering the clear heterogeneity, whether SSA has the similar efficacy in GEP-NET patients in Asian countries is worth investigation.

To address some of these issues in the present study, the expression of SSTR2 and SSTR5 was determined in a large cohort of GEP-NEN using immunohistochemistry, and findings were associated with clinicopathological variables and patient prognosis. In addition, the present study investigated the efficacy and safety of long-acting SSA octreotide LAR in Chinese GEP-NET patients by conducting a multicenter retrospective analysis on the data of 54 Chinese patients with unresectable, well-differentiated advanced or metastatic GEP-NET treated by octreotide LAR.

\section{Materials and methods}

Patient information. A total of 143 patients with histologically confirmed sporadic GEP-NEN at The First Affiliated Hospital,
Sun Yat-sen University (Guangzhou, China) from January 1995 to December 2014 were enrolled in the present study to determine the expression of SSTR2 and SSTR5. A total of 54 patients with advanced GEP-NET, who received octreotide LAR treatment in four centers across China with high-quality medical care between November 2009 and December 2015, were included in the present study to investigate the efficacy and safety of octreotide LAR in Chinese GEP-NET patients. Data from the following centers were included in the validation analysis: The First Affiliated Hospital, Sun Yat-Sen University $(n=31)$, Sun Yat-sen University Cancer Center (Guangzhou, China; n=12), Fudan University Shanghai Cancer Center (Shanghai, China; $\mathrm{n}=10$ ), Changzheng Hospital, Second Military Medical University (Shanghai, China; $n=1$ ). Electronic datasheets were provided for all participating centers. All de-identified data were reviewed and cross-checked for inconsistencies by YH Wang. Patient clinicopathological characteristics were summarized in Tables I and II.

A functional tumor was defined as overproducing a hormone such as 5-hydroxytryptamine, gastrin, glucagon, insulin, somatostatin and vasoactive intestinal peptide, which causes clinical symptoms. The pathology of each patient was reviewed according to the latest World Health Organization classification of tumors of the digestive system (19). Tumor-Node-Metastasis (TNM) stage was adopted according to the European Neuroendocrine Tumor Society Consensus Guidelines (20,21). Treatment responses were evaluated according to Response Evaluation Criteria in Solid Tumors (RECIST, version 1.1) (22).

The study was conducted in accordance with Declaration of Helsinki and in compliance with good clinical practice guidelines. The trial protocol was approved by the institutional review board of each institution. Written informed consent was obtained from each patient.

IHC. SSTR2 and SSTR5 IHC stains were performed in all 143 cases. Sections of tumor specimens (4- $\mu \mathrm{m}$ thick) from formalin-fixed paraffin-embedded sections were used for IHC examinations. The slides were dewaxed with xylene and rehydrated in a graded series of ethanol. Heat-induced epitope retrieval was performed using a microwave oven at $600 \mathrm{~W}$ for $30 \mathrm{~min}$ in preheated $10 \mathrm{mmol} / 1$ citric acid (pH 6.0). Endogenous peroxidase activity was blocked by incubating the slides in $3 \%$ hydrogen peroxide for $20 \mathrm{~min}$ at room temperature. The slides were transferred to phosphate-buffered saline and subsequently incubated at $4^{\circ} \mathrm{C}$ with rabbit monoclonal anti-SSTR2 (1:100; ab134152; Epitomics, Burlingame, CA, USA) and anti-SSTR5 (1:100; ab109495; Epitomics) overnight at $4^{\circ} \mathrm{C}$. The following day, sections were incubated in secondary antibody (Real EnVision Detection kit, ready-to-use; K5007; Dako; Agilent Technologies, Inc., Santa Clara, CA, USA) for $1 \mathrm{~h}$ at room temperature. The substrate chromogen, 3,3'-diaminobenzidine, enabled visualization of the complex via a brown precipitate. Hematoxylin (blue) counterstaining enabled the visualization of the cell nuclei with a light microscope (4500; Olympus Corporation, Tokyo, Japan). Omission of primary antibody served as a negative control.

Histological interpretation. For evaluation of SSTR2 and SSTR5 immunopositivity, a scoring system standardized and proposed by Volante et al (6) was used, at is has been reported 
to have a good correlation with Octreoscan findings. The scoring system was as follows: 0 , absence of immunoreactivity; 1, pure cytoplasmic immunoreactivity, either focal or diffuse; 2 , membranous reactivity in $<50 \%$ of tumor cells, irrespective of the presence of cytoplasmic staining; and 3, circumferential membranous reactivity in $>50 \%$ of tumor cells, irrespective of the presence of cytoplasmic staining. Cases with a score of 2-3 were considered as positive, and 0-1 were considered as negative.

All slides were evaluated independently by two investigators (Y.L. and L.X.) who were blinded to the patient clinical data. Any discordant results were subsequently reviewed together to reach agreement or determine an average value for disputed sections.

Treatment. The 54 patients were administered octreotide LAR from a starting dose of 20-40 mg, administered by intramuscular injection once every 28 days, and the treatment continued until disease progression, evidenced by imaging or occurrence of adverse reactions that rendered further drug administration impossible. The treatment was suspended or the therapeutic dose was adjusted (increasing or reducing the dose, or shortening the interval between injections) depending on tumor control or functional symptoms (carcinoid syndrome and diarrhea) and the severity of adverse reactions. In the present study, there were six patients whose dose was increased to $30-40 \mathrm{mg}$ during the period of treatment, and a single patient's dose was increased to $60 \mathrm{mg}$, with the interval between injections being shortened to 21 days. The reasons for adjustment of therapeutic dose or interval of injections for the seven patients were exacerbation of the functional symptoms. Tumors in the chest, abdomen and pelvic cavity were measured prior to treatment and once every 4-12 weeks following treatment by using three-dimension spiral computed tomography or magnetic resonance imaging, and the size of tumor was evaluated by the imaging experts. The patient clinicopathological data, as well as the data of imaging examination following octreotide LAR treatment, were collected.

Efficacy and safety assessments. The primary study endpoint was TTP. The secondary endpoints included overall survival (OS), objective response rate (ORR) and stable disease (SD) rate. The adverse reactions were evaluated according to Common Terminology Criteria for Adverse Events (version 4.0) published by the U.S. National Cancer Institute (23).

Statistical analysis. SPSS version 16.0 software (SPSS, Inc., Chicago, IL, USA) was employed for statistical analysis of the data. Descriptive statistics of qualitative data such as patient's general data, positive expression rates, treatment evaluation and adverse reactions, were expressed as numbers and percentages. The results of SSTR2 and SSTR5 expression analysis were compared in terms of various clinicopathological data, including functional status, tumor site, grade, type and stage. Statistical evaluation was performed by means of the $\chi^{2}$ tests. OS and TTP analyses were performed using Kaplan-Meier survival plots and comparisons between groups were made with the log-rank test. ORR and SD rate were described using percentage, and $95 \%$ confidence intervals (CIs) were calculated. $\mathrm{P}<0.05$ was considered to indicate a statistically significant difference.
Table I. Clinicopathological characteristics of gastroenteropancreatic neuroendocrine neoplasm patients with somatostatin receptor immunohistochemical detection.

\begin{tabular}{|c|c|c|}
\hline $\begin{array}{l}\text { Demographic and clinical } \\
\text { characteristics }(n=143)\end{array}$ & $\mathrm{n}$ & $\%$ \\
\hline \multicolumn{3}{|l|}{ Gender $^{\mathrm{a}}$} \\
\hline Male & 87 & 60.8 \\
\hline Female & 56 & 39.2 \\
\hline \multicolumn{3}{|l|}{ Age at diagnosis (years) } \\
\hline$\leq 50$ & 71 & 49.7 \\
\hline$>50$ & 72 & 50.3 \\
\hline Median (range) & $51(18-85)$ & \\
\hline \multicolumn{3}{|l|}{ Functional status } \\
\hline Nonfunctional & 113 & 79.0 \\
\hline Functional & 30 & 21.0 \\
\hline Insulinoma & 24 & 16.8 \\
\hline Vasoactive intestinal polypeptidoma & 4 & 2.8 \\
\hline Somatostatinoma & 1 & 0.7 \\
\hline Carcinoid syndrome & 1 & 0.7 \\
\hline \multicolumn{3}{|l|}{ Tumor location } \\
\hline Gastrointestinal tract & 79 & 55.2 \\
\hline Rectum & 34 & 23.8 \\
\hline Stomach & 19 & 13.3 \\
\hline Duodenum & 15 & 10.5 \\
\hline Jejunum/ileum & 7 & 4.9 \\
\hline Appendix & 4 & 2.8 \\
\hline Pancreas & 64 & 44.8 \\
\hline \multicolumn{3}{|l|}{ Tumor grade } \\
\hline G1 & 69 & 48.3 \\
\hline G2 & 39 & 27.3 \\
\hline G3 & 35 & 24.5 \\
\hline \multicolumn{3}{|l|}{ Tumor type } \\
\hline NET & 110 & 76.9 \\
\hline NET G1 & 69 & 48.3 \\
\hline NET G2 & 39 & 27.3 \\
\hline NET G3 & 2 & 1.4 \\
\hline NEC & 31 & 21.7 \\
\hline MANEC & 2 & 1.4 \\
\hline \multicolumn{3}{|l|}{ Tumor stage } \\
\hline I & 43 & 30.1 \\
\hline II & 28 & 19.6 \\
\hline III & 16 & 11.2 \\
\hline IV & 56 & 39.2 \\
\hline
\end{tabular}

${ }^{\mathrm{a} M a l e: f e m a l e, ~ 1.55: 1 . ~ N E T, ~ n e u r o e n d o c r i n e ~ t u m o r ; ~ N E C, ~ n e u r o e n d o-~}$ crine carcinoma; MANEC, mixed adenoneuroendocrine carcinoma.

\section{Results}

Immunohistochemical expression of SSTR2 and SSTR5 in $G E P-N E N$. As shown in Fig. 1, SSTR2 was positively immunostained in the membrane of tumor cells, and varied from 
Table II. Clinicopathological characteristics of gastroenteropancreatic neuroendocrine tumor patients with octreotide long-acting release treatment.

\section{Demographic and clinical}

characteristics $(n=54)$

$\mathrm{n} \quad \%$

Gender $^{\mathrm{a}}$

Male

Female

Age at diagnosis (years)

$\leq 50$

$>50$

Median (range)

$50(18-72)$

\section{ECOG PS}

0

1

2

Functional status

Nonfunctional

Functional

Vasoactive intestinal polypeptidoma

Carcinoid syndrome

Gastrinoma

Insulinoma

Tumor location

Gastrointestinal tract

Rectum

Jejunum/ileum

Duodenum

Pancreas

$\mathrm{Ki67}$ index (\%)

$\leq 2$

3-10

$>10$

Tumor grade

G1

G2

G3

Tumor stage

IV

Combined treatment

Monotherapy

With targeted drug therapy

With interventional therapy

With chemotherapy

With palliative surgery

With $>2$ therapies

Previous treatment

None

Surgical therapy

Targeted drug therapy

Interventional therapy

Chemotherapy

$>2$ therapies
Table II. Continued.

Demographic and clinical characteristics $(n=54)$ $\mathrm{n}$ $\%$

\section{SSTR2 expression ${ }^{\mathrm{b}}$}

Positive

86.4

Negative

SSTR5 expression ${ }^{\mathrm{b}}$

Positive

18

81.8

Negative

4

18.2

${ }^{a}$ Male:female, 1.45:1. ' In total, 22 cases for both SSTR2 and SSTR5 expression were observed. ECOG PS, Eastern Cooperative Oncology Group Performance Status; SSTR, somatostatin receptor.
29.6 weak-incomplete to strong-complete staining. The overall expression rate of SSTR2 was $67.8 \%$ (97/143). Membranous SSTR5 immunopositivity was noted in 56.6\% (81/143) of tumors. No nuclear immunostaining was observed.

Association of SSTR2 and SSTR5 expression with clinicopathological variables. SSTR2 expression was increased in tumors with hormonal syndrome $(\mathrm{P}=0.041)$. Patients with pancreatic tumors had a significantly increased SSTR2 expression compared with gastrointestinal (GI) tumors (79.7 vs. 58.2\%; $\mathrm{P}=0.006$ ). Poorly differentiated tumors [G3 tumors and neuroendocrine carcinoma (NEC) + mixed adenoneuroendocrine carcinoma (MANEC)] had lower SSTR2 expression compared with well- and moderately-differentiated tumors [G1, G2 tumors and neuroendocrine tumor (NET); P<0.001]. The expression rate of SSTR2 in tumors of stage I and II was $77.5 \%$, which was markedly increased compared with tumors of stage III and IV (58.3\%; P=0.014). Similarly, SSTR5 was significantly increased in pancreatic and well-differentiated tumors compared with in gastrointestinal and poorly differentiated tumors $(\mathrm{P}=0.022, \mathrm{P}=0.008$ and $\mathrm{P}=0.002$, respectively). The expression rates and statistical data are summarized in Table III.

Association of SSTR2 and SSTR5 expression with survival. A total of 116/143 patients received long-term follow up with a median duration of 3.36 years (range, 0.02-15.05 years). At the final follow-up, 36 patients $(31.0 \%)$ had succumbed to the disease. The major causes of mortality were tumor-associated $(34 / 36 ; 94.4 \%)$, and treatment-associated adverse events $(2 / 36$; $5.6 \%$; both succumbed from surgical complications). Only NEN-associated mortalities were considered as events for survival analysis.

Kaplan-Meier survival curves revealed that the median OS time of patients with positive expression of SSTR2 was not reached (NR), while patients with negative expression had a median OS of 3.48 years, which demonstrated a statistically significant difference $\left(\chi^{2}=8.758, P=0.003\right)$. Similarly, SSTR5 positive expression also predicted improved survival compared with negative expression (the median OS times were NR and 7.22 years, respectively; $\chi^{2}=6.396, P=0.011$ ) (Fig. 2). 

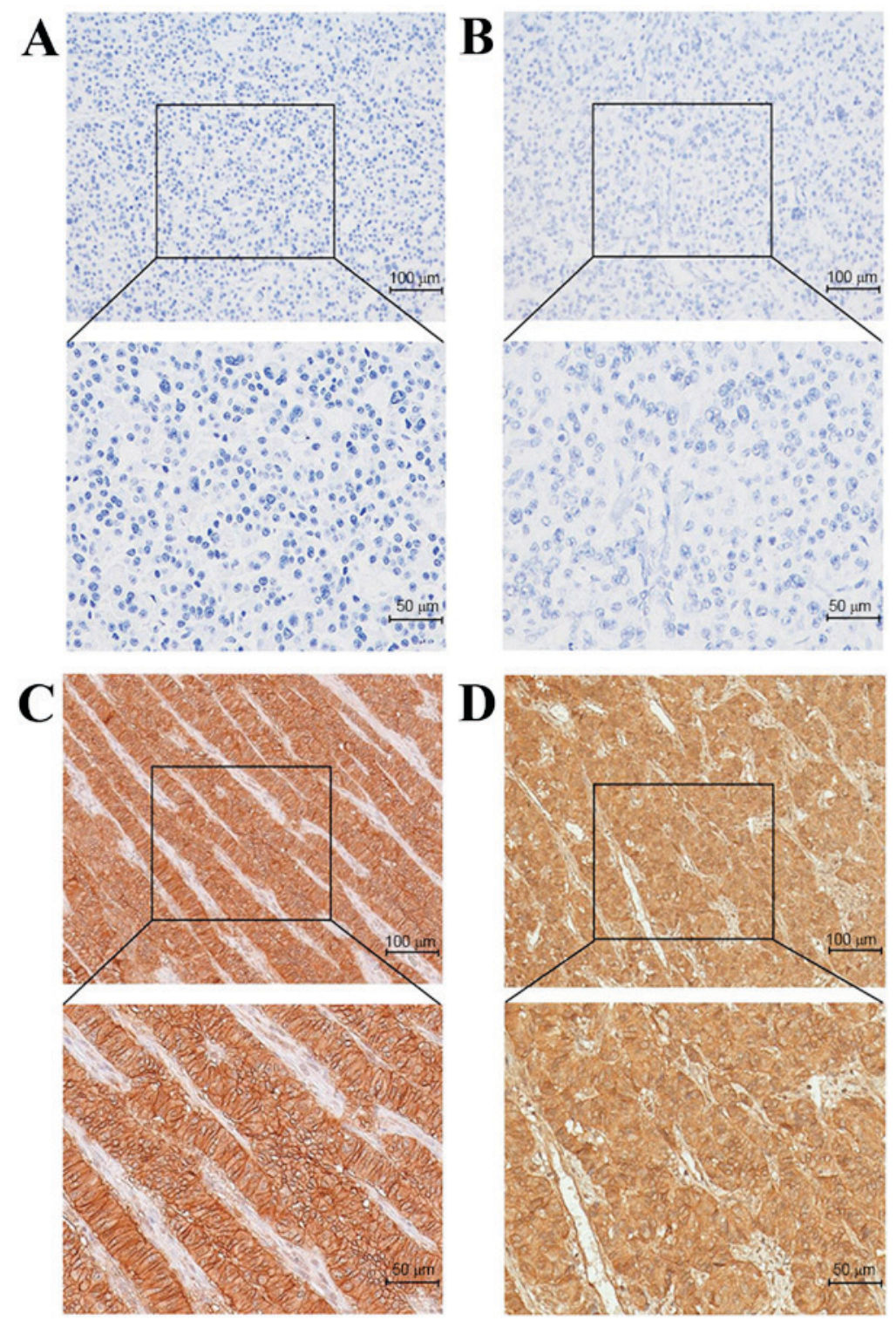

Figure 1. Immunohistochemical staining of SSTR2 and SSTR5 in gastroenteropancreatic neuroendocrine neoplasm (using the EnVision method). (A) Pancreatic NET, G2, SSTR2-negative staining. (B) Pancreatic NET, G2, SSTR5-negative staining. (C) Pancreatic NET, G2, strong SSTR2-positive staining. (D) Pancreatic NET, G2, strong SSTR5-positive staining. For each panel: Upper panel magnification, x20; lower panel magnification, x40. SSTR, somatostatin receptor; NET, neuroendocrine tumor.

Efficacy assessment. All 54 patients that received octreotide LAR were followed up for a period of 3.2-164.5 months, with a median follow-up period of 31.8 months. By the conclusion of follow-up, 11 of the patients died of progressive disease (PD) and 26 of the patients were still receiving octreotide LAR treatment. The median OS was not reached and the median TTP was 20.2 months (95\% CI, 13.9-26.5\%) (Fig. 3). Imaging evaluation was performed for all patients according to RECIST, and three patients achieved partial remission (PR), with the ORR being 5.6\% (95\% CI, 0.0-11.7\%). A total of 43 patients achieved SD, with the SD rate being $79.6 \%$ (95\% CI, 68.9-90.4\%) and 8 patients demonstrated PD. At the conclusion of follow-up, there were still three patients achieving PR, 26 patients achieving SD and 25 patients demonstrating PD.

The median TTP in all 54 patients treated with octreotide LAR was not associated with the patient's functional status, tumor site, Ki67 index and whether or not they received other anti-tumor therapy prior to octreotide LAR treatment or combined therapy $(\mathrm{P}=0.116, \mathrm{P}=0.665, \mathrm{P}=0.512, \mathrm{P}=0.256$ and $\mathrm{P}=0.817$, respectively). No associations between the expression of SSTR 2 and SSTR5 and median TTP were evident $(\mathrm{P}=0.352$ and 0.575 , respectively; Table IV).

Safety assessment. A total of 14/54 (25.9\%) patients experienced adverse drug reactions during the period of octreotide LAR treatment, and the most common grade 1-2 adverse events (AEs) were diarrhea (16.7\%), abdominal distension (7.4\%), abdominal pain $(7.4 \%)$ and elevation of blood glucose (1.9\%). Octreotide LAR-associated AEs occurred 1-4 weeks following administration of the drug, primarily in the initial one or two weeks. All of the above AEs were relieved or remedied following symptomatic treatment. No serious adverse events (SAE) were observed during the present study. None of the patients required dose reduction or drug withdrawal due to $\mathrm{AE}$. 
Table III. Association of SSTR2 and SSTR5 expression with clinicopathological variables ( $\mathrm{n}=143)$.

\begin{tabular}{|c|c|c|c|c|c|c|c|}
\hline Characteristic & $\mathrm{n}$ & SSTR2 positive, $\mathrm{n}(\%)$ & $\chi^{2}$ value & P-value & SSTR5 positive, n (\%) & $\chi^{2}$ value & P-value \\
\hline Functional status & & & 4.181 & 0.041 & & 0.692 & 0.406 \\
\hline Nonfunctional & 113 & $72(63.7)$ & & & $62(54.9)$ & & \\
\hline Functional & 30 & $25(83.3)$ & & & $19(63.3)$ & & \\
\hline Site & & & 7.462 & 0.006 & & 5.245 & 0.022 \\
\hline Gastrointestinal tract & 79 & $46(58.2)$ & & & $38(48.1)$ & & \\
\hline Pancreas & 64 & $51(79.7)$ & & & $43(67.2)$ & & \\
\hline Tumor grade & & & 20.330 & $<0.001$ & & 9.570 & 0.008 \\
\hline G1 & 69 & $55(79.7)$ & & & $45(65.2)$ & & \\
\hline G2 & 39 & $29(74.4)$ & & & $24(61.5)$ & & \\
\hline G3 & 35 & $13(37.1)$ & & & $12(34.3)$ & & \\
\hline Tumor type & & & 23.400 & $<0.001$ & & 9.492 & 0.002 \\
\hline NET & 110 & $86(78.2)$ & & & $70(63.6)$ & & \\
\hline NEC+MANEC & 33 & $11(33.3)$ & & & $11(33.3)$ & & \\
\hline Tumor stage & & & 5.996 & 0.014 & & 0.070 & 0.792 \\
\hline $\mathrm{I}+\mathrm{II}$ & 71 & $55(77.5)$ & & & $41(57.7)$ & & \\
\hline III+IV & 72 & $42(58.3)$ & & & $40(55.6)$ & & \\
\hline
\end{tabular}

SSTR, somatostatin receptor; NET, neuroendocrine tumor; NEC, neuroendocrine carcinoma; MANEC, mixed adenoneuroendocrine carcinoma.
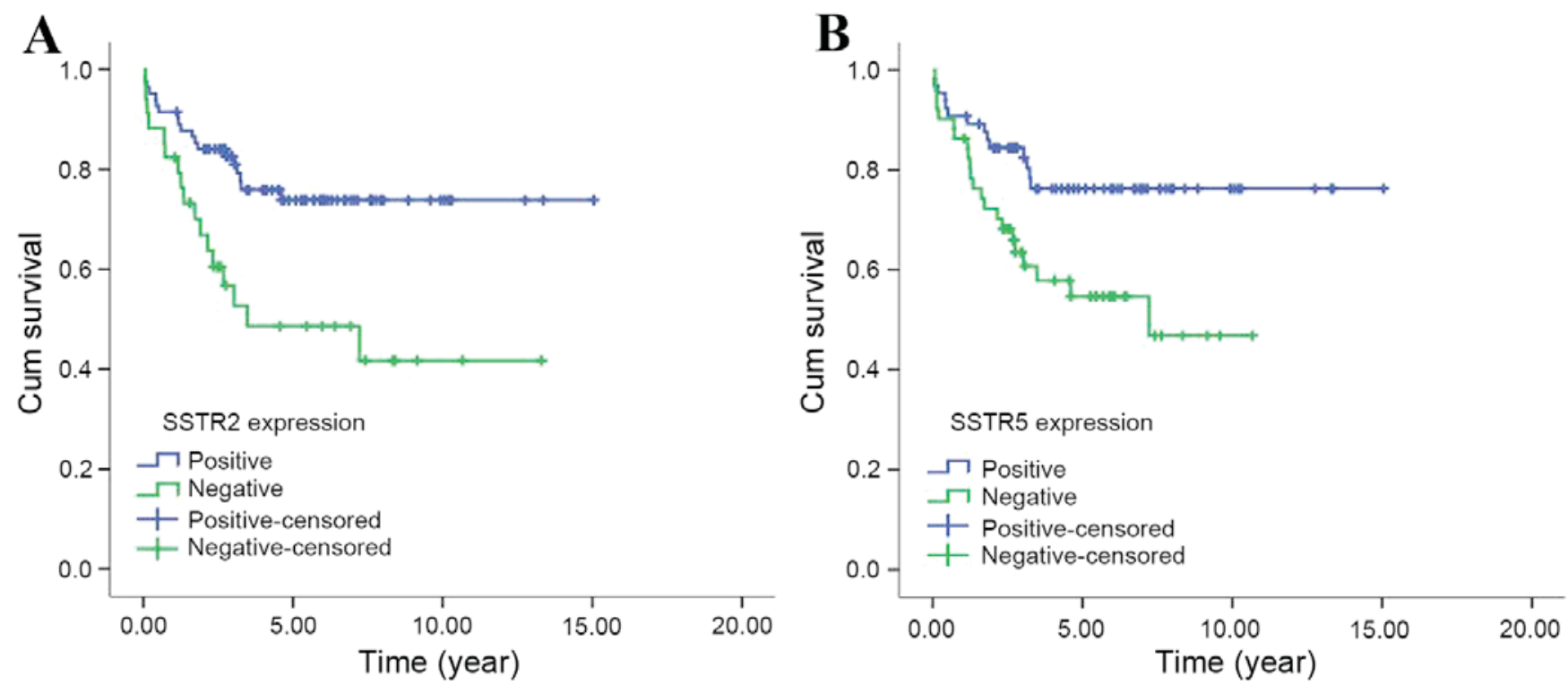

Figure 2. Kaplan-Meier curves. Overall survival by (A) SSTR2 and (B) SSTR5 expression in gastroenteropancreatic neuroendocrine neoplasm. SSTR, somatostatin receptor; Cum, cumulative.

\section{Discussion}

The wide expression of SSTRs in neuroendocrine tumors has been investigated by various methods (2-4). Immunohistochemistry appears to be a reliable and reproducible technique to detect the SSTRs in GEP-NEN with clear advantages, including low cost, easy operation and allowing the SSTR profile determination of GEP-NEN in the clinical setting (24). The expression rates of SSTR2 and SSTR5 with immunohistochemistry in GEP-NEN have been reported in previous studies to be within the range of $60-93$ and $38-83 \%$, respectively $(2,5,24-28)$. In the present study, it was observed that the overall expression rates of SSTR2 and SSTR5 were 67.8 and $56.6 \%$, comprising a total of 143 samples from GEP-NEN patients, which was comparable to previous studies.

Srirajaskanthan et al (29) reported that SSTR2 and SSTR5 expression were inversely correlated with neuroendocrine tumor grade. Low to intermediate-grade tumors, which were also well-differentiated, had increased SSTR expression compared with high-grade tumors $(\mathrm{P}<0.005)(29)$. In line with previous findings, the present study demonstrated a gradual decline in SSTR2 and SSTR5 expression of 
Table IV. Time to progression and its association with the sub-groups $(\mathrm{n}=54)$.

\begin{tabular}{|c|c|c|c|c|c|}
\hline Characteristics & $\mathrm{n}$ & Median (months) & $95 \% \mathrm{CI}$ & $\chi^{2}$ value & P-value \\
\hline Patients with octreotide LAR treatment & 54 & 20.2 & $13.9-26.5$ & & \\
\hline Functional status & & & & 2.474 & 0.116 \\
\hline Non-functional & 41 & 17.5 & $11.0-23.9$ & & \\
\hline Functional & 13 & 67.9 & $\mathrm{NC}$ & & \\
\hline Tumor site & & & & 0.188 & 0.665 \\
\hline Gastrointestinal tract & 13 & 17.5 & $0.0-43.7$ & & \\
\hline Pancreas & 41 & 20.2 & $12.0-28.4$ & & \\
\hline $\mathrm{Ki}-67$ index $(\%)$ & & & & 1.340 & 0.512 \\
\hline$\leq 2$ & 11 & 67.9 & $\mathrm{NC}$ & & \\
\hline $3-10$ & 33 & 20.6 & $15.0-26.2$ & & \\
\hline$>10$ & 10 & 10.9 & $3.3-18.5$ & & \\
\hline Previous treatment & & & & 1.288 & 0.256 \\
\hline No & 16 & NR & $\mathrm{NC}$ & & \\
\hline Yes & 38 & 16.0 & $5.6-26.5$ & & \\
\hline Combined therapy & & & & 0.053 & 0.817 \\
\hline No & 31 & 17.5 & $4.5-30.5$ & & \\
\hline Yes & 23 & 20.2 & $10.9-29.5$ & & \\
\hline SSTR2 expression ${ }^{a}$ & & & & 0.867 & 0.352 \\
\hline Positive & 19 & 20.6 & $10.5-30.7$ & & \\
\hline Negative & 3 & 9.4 & $\mathrm{NC}$ & & \\
\hline SSTR5 expression ${ }^{a}$ & & & & 0.314 & 0.575 \\
\hline Positive & 18 & 16.0 & $6.4-25.7$ & & \\
\hline Negative & 4 & NR & $\mathrm{NC}$ & & \\
\hline
\end{tabular}

'In total, 22 cases for both SSTR2 and SSTR5 expression were observed. CI, confidence interval; NR, not reached; NC, not computable; LAR, long-acting release; SSTR, somatostatin receptor.
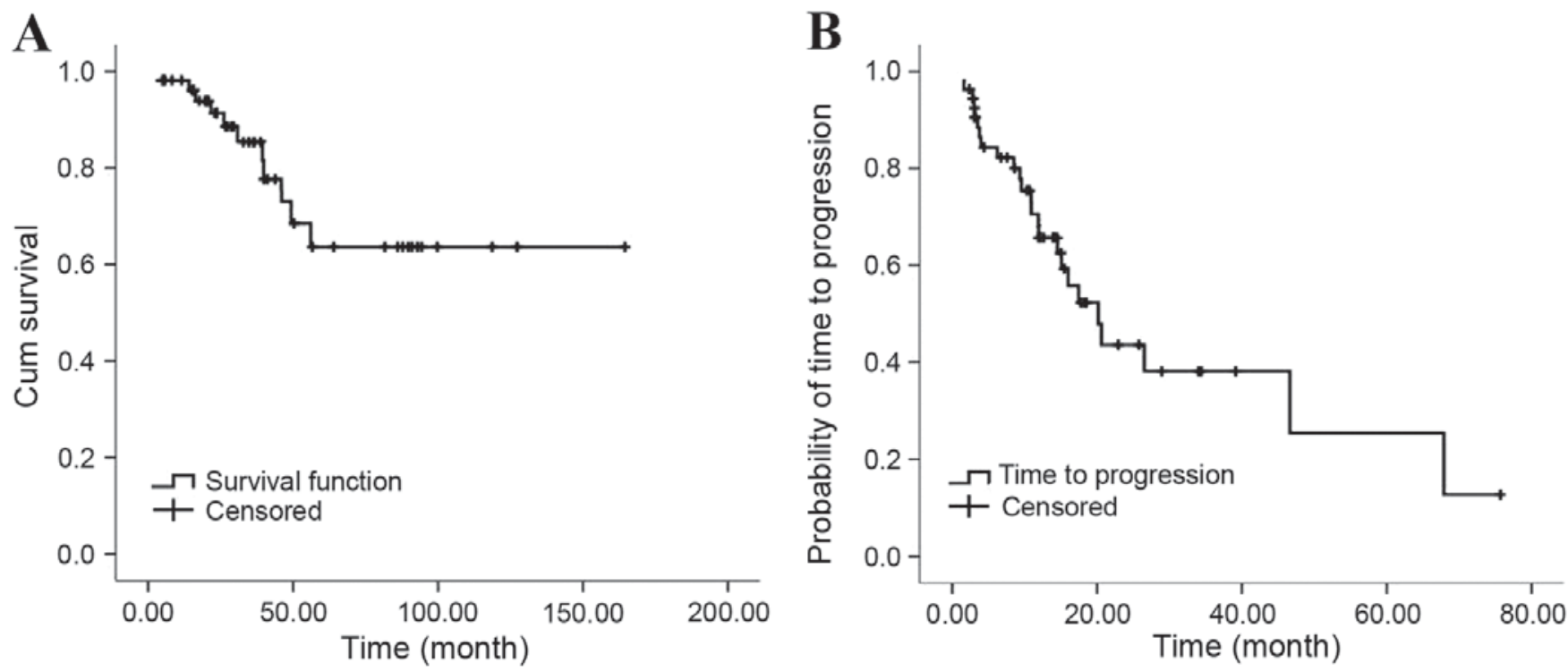

Figure 3. Kaplan-Meier curves. (A) Overall survival and (B) time to progression in gastroenteropancreatic neuroendocrine tumor patients with octreotide long-acting release treatment. Cum, cumulative.

well-(G1, G2 and NET) and poorly-differentiated tumors (G3 and NEC+MANEC; $\mathrm{P}<0.001, \mathrm{P}<0.001, \mathrm{P}=0.008$,
$\mathrm{P}=0.002$, respectively). The present study also observed that SSTR2 and SSTR5 were significantly more likely to be 
expressed in pancreatic tumors than GI tumors $(\mathrm{P}=0.006$ and 0.022 , respectively). In addition, SSTR 2 expression was significantly increased in tumors with hormonal syndrome and TNM stage I and II ( $\mathrm{P}=0.041$ and 0.014 , respectively); however, SSTR5 was not. These data are inconsistent with the results of previous studies $(2,25,30)$, which revealed that no association was observed between SSTR expression and tumor location, functional status and TNM stage. However, these previous studies mainly focused on particular types of GEP-NENs, including well-differentiated endocrine tumors or a single site of tumor (pancreas). According to the above results in this study, it was observed that SSTR subtype expression demonstrates marked heterogeneity and differences in tumor sites and differentiation, and a decrease in SSTR2 and SSTR5 expression with increasing malignancy in GEP-NEN.

Previous studies investigating SSTR subtype expression as a prognostic factor have shown conflicting results. In a study of 60 patients with GEP-NEN, Kaemmerer et al (31) showed that positive staining for SSTR2 $(n=54)$ was associated with significantly longer OS as compared to negative staining $(\mathrm{n}=6$; median OS, 49.5 vs. 16.5 months; $\mathrm{P}<0.001)$. Corleto et al (32) observed a significantly better survival rate in patients with well-differentiated neuroendocrine tumors expressing SSTR2, SSTR5 and Ki-67<2\% simultaneously. However, Papotti et al (2) reported no statistically significant correlation between SSTR subtype expression and clinical outcome in 54 cases. This discrepancy may be due to the small number of a negligible SSTR2 expression cases, and the differences in tumor origin and differentiation. Although the present results concerning the association between SSTR expression and survival were inconsistent, the current study indicated that patients with SSTR2 and SSTR5 positive expression had an improved prognosis.

SSAs have been proved in many clinical studies to be able to inhibit the secretion of tumor-producing hormones by binding with SSTRs on the surface of neuroendocrine neoplasm cells. Placebo controlled PROMID and CLARINET studies have further discovered that SSAs have anti-tumor activity along with inhibiting hormone secretion $(11,12)$. The present investigation conducted a multicenter retrospective study of octreotide LAR in the treatment of 54 Chinese patients with unresectable, well-differentiated advanced or metastatic GEP-NETs, finding that the overall median TTP was 20.2 months (95\% CI, 13.9-26.5), with an ORR of 5.6\% and an SD rate of $79.6 \%$. Analysis of the subgroups showed that differences in the median TTP were not statistically significant regarding the primary site of tumor (GI tract and pancreas) and functional status ( $\mathrm{P}=0.665$ and $\mathrm{P}=0.116$, respectively). The above results were similar to the results of the studies in the Western population, indicating that octreotide LAR is effective in Chinese GEN-NET patients, regardless of whether the primary site is GI tract or pancreas and whether the tumor is functional or not.

A retrospective study comprising 43 patients with pancreatic NET treated with octreotide LAR conducted by Jann et al (33) revealed that patients with a Ki67 $\leq 10 \%$ showed a longer median TTP than those with a Ki67 $>10 \%$. In the present study, although no statistically significant difference was observed $(\mathrm{P}=0.512)$, a tendency for octreotide LAR to show improved efficacy in patients with Ki67 $\leq 10 \%$ (the median TTP in patients with Ki67 $\leq 2 \%$, Ki67 of 3-10\% and Ki67 >10\% was 67.9, 20.6 and 10.9 months, respectively) was identified. The above results suggested that patients with lower proliferation index appear to have a longer TTP and may be candidates for octreotide LAR treatment.

In the present study, the therapeutic dose was increased or the interval of injection was shortened for $7 / 54$ patients during the period of octreotide LAR treatment, due to exacerbation of the functional symptoms, and the patient symptoms were thus improved. Previous studies showed that increases in the dose or frequency of SSA may be considered for patients with poor control of symptoms and tumors, particularly in cases where disease was previously stabilized at a lower dose (34-37). Therefore, efficacy can be obtained again by adjusting the dose of SSA or the interval of treatment in clinical practice.

To the best of our knowledge, there have been few studies focused on the predictive value of SSTR immunohistochemistry in determining the treatment response to SSA. In the present study, the differences between SSTR subtype expression and median TTP were not statistically significant $(\mathrm{P}=0.352$ and $\mathrm{P}=0.575$, respectively). Such an association was limited in the present study because of heterogeneous biological behavior of the disease and a small number of patients with SSTR subtype detection (22 patients). Large clinical trials should be designed to validate the role of somatostatin receptor immunohistochemical profile in the prediction of clinical response.

SSA is a therapeutic approach that has much fewer side effects and higher safety than targeted drugs $(18,38)$ or cytotoxic drugs $(39,40)$. In the PROMID study, 11 (12.9\%) of the 85 patients experienced SAE, with the common adverse reactions in the octreotide LAR group being diarrhea and abdominal distension, and five of the patients discontinued the treatment due to AE (11). In the CLARINET study, 50\% of the 101 patients in the lanreotide group experienced AEs, and three $(3.0 \%)$ of the patients experienced SAE, one of whom withdrew from the study due to AE (12). Adverse reactions observed in the present study were diarrhea, abdominal distension and abdominal pain, being similar to those in the aforementioned studies. However, octreotide LAR showed improved safety in Chinese patients on the whole, with a lower incidence (25.9\%) of AE, and none of the patients experienced an SAE or required dose reduction or drug withdrawal due to AE.

In conclusion, the present study demonstrates that SSTR2 and SSTR5 are heterogeneously expressed in GEP-NEN with different tumor sites and differentiation. Both markers could serve as potential prognostic factors to predict survival. Furthermore, although the present retrospective study included only 54 cases, the efficacy and safety of octreotide LAR in China was investigated for the first time. It was observed that octreotide LAR is effective in the treatment of Chinese patients with well-differentiated advanced GEP-NET, with a low incidence of adverse reactions.

\section{Acknowledgements}

The authors are grateful to Dr Yuan Gao (Department of Gastroenterology, The First Affiliated Hospital, Sun Yat-Sen University, Guangzhou, China) for skillful writing assistance. 


\section{References}

1. Modlin IM, Oberg K, Chung DC, Jensen RT, de Herder WW, Thakker RV, Caplin M, Delle FAVE G, Kaltsas GA, Krenning EP, et al: Gastroenteropancreatic neuroendocrine tumours. Lancet Oncol 9: 61-72, 2008.

2. Papotti M, Bongiovanni M, Volante M, Allìa E, Landolfi S, Helboe L, Schindler M, Cole SL and Bussolati G: Expression of somatostatin receptor types 1-5 in 81 cases of gastrointestinal and pancreatic endocrine tumors. A correlative immunohistochemical and reverse-transcriptase polymerase chain reaction analysis. Virchows Arch 440: 461-475, 2002

3. Kulaksiz H, Eissele R, Rössler D, Schulz S, Höllt V, Cetin Y and Arnold R: Identification of somatostatin receptor subtypes $1,2 \mathrm{a}, 3$ and 5 in neuroendocrine tumours with subtype specific antibodies. Gut 50: 52-60, 2002.

4. Korner M, Eltschinger V, Waser B, Schonbrunn A and Reubi JC: Value of immunohistochemistry for somatostatin receptor subtype sst2a in cancer tissues: Lessons from the comparison of anti-sst2a antibodies with somatostatin receptor autoradiography. Am J Surg Pathol 29: 1642-1651, 2005.

5. Zamora V, Cabanne A, Salanova R, Bestani C, Domenichini E, Marmissolle F, Giacomi N, O'Connor J, Méndez G and Roca E; Buenos Aires and La Plata Argentina Argentum Working Group: Immunohistochemical expression of somatostatin receptors in digestive endocrine tumours. Dig Liver Dis 42: 220-225, 2010.

6. Volante M, Brizzi MP, Faggiano A, La Rosa S, Rapa I, Ferrero A, Mansueto G, Righi L, Garancini S, Capella C, et al: Somatostatin receptor type 2a immunohistochemistry in neuroendocrine tumors: A proposal of scoring system correlated with somatostatin receptor scintigraphy. Mod Pathol 20: 1172-1182, 2007.

7. Hofland LJ and Lamberts SW: The pathophysiological consequences of somatostatin receptor internalization and resistance. Endocr Rev 24: 28-47, 2003

8. Oberg KE, Reubi JC, Kwekkeboom DJ and Krenning EP: Role of somatostatins in gastroenteropancreatic neuroendocrine tumor development and therapy. Gastroenterology 139: 742-753, 753.e1, 2010

9. Oberg K: Future aspects of somatostatin-receptor-mediated therapy. Neuroendocrinology 80 (Suppl 1): S57-S61, 2004.

10. Appetecchia M and Baldelli R: Somatostatin analogues in the treatment of gastroenteropancreatic neuroendocrine tumours, current aspects and new perspectives. J Exp Clin Cancer Res 29: 19, 2010.

11. Rinke A, Müller HH, Schade-Brittinger C, Klose KJ, Barth P, Wied M, Mayer C, Aminossadati B, Pape UF, Bläker M, et al: Placebo-controlled, double-blind, prospective, randomized study on the effect of octreotide lar in the control of tumor growth in patients with metastatic neuroendocrine midgut tumors: A report from the promid study group. J Clin Oncol 27 : 4656-4663, 2009.

12. Caplin ME, Pavel M, Ćwikła JB, Phan AT, Raderer M, Sedláčková E, Cadiot G, Wolin EM, Capdevila J, Wall L, et al: Lanreotide in metastatic enteropancreatic neuroendocrine tumors. N Engl J Med 371: 224-233, 2014.

13. Lim T, Lee J, Kim JJ, Lee JK, Lee KT, Kim YH, Kim KW, Kim S, Sohn TS, Choi DW, et al: Gastroenteropancreatic neuroendocrine tumors: Incidence and treatment outcome in a single institution in korea. Asia Pac J Clin Oncol 7: 293-299, 2011.

14. Hauso O, Gustafsson BI, Kidd M, Waldum HL, Drozdov I, Chan AK and Modlin IM: Neuroendocrine tumor epidemiology: Contrasting norway and north america. Cancer 113: 2655-2664, 2008.

15. Yao JC, Hassan M, Phan A, Dagohoy C, Leary C, Mares JE, Abdalla EK, Fleming JB, Vauthey JN, Rashid A and Evans DB: One hundred years after 'carcinoid': Epidemiology of and prognostic factors for neuroendocrine tumors in 35,825 cases in the united states. J Clin Oncol 26: 3063-3072, 2008

16. Wang YH, Lin Y, Xue L, Wang JH, Chen MH and Chen J: Relationship between clinical characteristics and survival of gastroenteropancreatic neuroendocrine neoplasms: A single-institution analysis (1995-2012) in south china. BMC Endocr Disord 12: 30, 2012.

17. Ito T, Okusaka T, Nishida T, Yamao K, Igarashi H, Morizane C, Kondo S, Mizuno N, Hara K, Sawaki A, et al: Phase ii study of sunitinib in japanese patients with unresectable or metastatic, well-differentiated pancreatic neuroendocrine tumor. Invest New Drugs 31: 1265-1274, 2013.
18. Raymond E, Dahan L, Raoul JL, Bang YJ, Borbath I, Lombard-Bohas C, Valle J, Metrakos P, Smith D, Vinik A, et al: Sunitinib malate for the treatment of pancreatic neuroendocrine tumors. N Engl J Med 364: 501-513, 2011.

19. Bosman F, Carneiro F, Hruban R and Theise N: Who classification of tumours of the digestive system. 4th edition. IARC Press, PA, 2010

20. Rindi G, Klöppel G, Alhman H, Caplin M, Couvelard A, de Herder WW, Erikssson B, Falchetti A, Falconi M, Komminoth $\mathrm{P}$, et al: Tnm staging of foregut (neuro)endocrine tumors: A consensus proposal including a grading system. Virchows Arch 449: 395-401, 2006.

21. Rindi G, Klöppel G, Couvelard A, Komminoth P, Körner M, Lopes JM, McNicol AM, Nilsson O, Perren A, Scarpa A, et al: Tnm staging of midgut and hindgut (neuro) endocrine tumors: A consensus proposal including a grading system. Virchows Arch 451: 757-762, 2007.

22. Eisenhauer EA, Therasse P, Bogaerts J, Schwartz LH, Sargent D, Ford R, Dancey J, Arbuck S, Gwyther S, Mooney M, et al: New response evaluation criteria in solid tumours: Revised RECIST guideline (version 1.1). Eur J Cancer 45: 228-247, 2009.

23. Chen AP, Setser A, Anadkat MJ, Cotliar J, Olsen EA, Garden BC and Lacouture ME: Grading dermatologic adverse events of cancer treatments: The common terminology criteria for adverse events version 4.0. J Am Acad Dermatol 67: 1025-1039, 2012

24. Diakatou E, Kaltsas G, Tzivras M, Kanakis G, Papaliodi E and Kontogeorgos G: Somatostatin and dopamine receptor profile of gastroenteropancreatic neuroendocrine tumors: An immunohistochemical study. Endocr Pathol 22: 24-30, 2011.

25. van Adrichem RC, Kamp K, van Deurzen CH, Biermann K, Feelders RA, Franssen GJ, Kwekkeboom DJ, Hofland LJ and de Herder WW: Is there an additional value of somatostatin receptor subtype 2 a immunohistochemistry over somatostatin receptor scintigraphy uptake in predicting gastroenteropancreatic neuroendocrine tumor response? Neuroendocrinology 103: $560-566,2016$.

26. Sclafani F, Carnaghi C, Di Tommaso L, Rodari M, Destro A, Rimassa L, Giordano L, Chiti A, Roncalli M and Santoro A: Detection of somatostatin receptor subtypes 2 and 5 by somatostatin receptor scintigraphy and immunohistochemistry: Clinical implications in the diagnostic and therapeutic management of gastroenteropancreatic neuroendocrine tumors. Tumori 97: 620-628, 2011

27. Yerci O, Sehitoglu I, Ugras N, Cubukcu E, Yuce S, Bedir R and Cure E: Somatostatin receptor 2 and 5 expressions in gastroenteropancreatic neuroendocrine tumors in turkey. Asian Pac J Cancer Prev 16: 4377-4381, 2015.

28. Nasir A, Stridsberg M, Strosberg J, Su PH, Livingston S, Malik HA, Kelley ST, Centeno BA, Coppola D, Malafa ME, et al: Somatostatin receptor profiling in hepatic metastases from small intestinal and pancreatic neuroendocrine neoplasms: Immunohistochemical approach with potential clinical utility. Cancer Control 13: 52-60, 2006.

29. Srirajaskanthan R, Watkins J, Marelli L, Khan K and Caplin ME: Expression of somatostatin and dopamine 2 receptors in neuroendocrine tumours and the potential role for new biotherapies. Neuroendocrinology 89: 308-314, 2009.

30. Okuwaki K, Kida M, Mikami T, Yamauchi H, Imaizumi H, Miyazawa S, Iwai T, Takezawa M, Saegusa M, Watanabe M and Koizumi W: Clinicopathologic characteristics of pancreatic neuroendocrine tumors and relation of somatostatin receptor type 2a to outcomes. Cancer 119: 4094-4102, 2013.

31. Kaemmerer D, Trager T, Hoffmeister M, Sipos B, Hommann M, Sänger J, Schulz S and Lupp A: Inverse expression of somatostatin and cxcr4 chemokine receptors in gastroenteropancreatic neuroendocrine neoplasms of different malignancy. Oncotarget 6: 27566-27579, 2015.

32. Corleto VD, Falconi M, Panzuto F, Milione M, De Luca O, Perri P, Cannizzaro R, Bordi C, Pederzoli P, Scarpa A and Delle Fave G: Somatostatin receptor subtypes 2 and 5 are associated with better survival in well-differentiated endocrine carcinomas. Neuroendocrinology 89: 223-230, 2009.

33. Jann H, Denecke T, Koch M, Pape UF, Wiedenmann B and Pavel M: Impact of octreotide long-acting release on tumour growth control as a first-line treatment in neuroendocrine tumours of pancreatic origin. Neuroendocrinology 98: 137-143, 2013.

34. Broder MS, Beenhouwer D, Strosberg JR, Neary MP and Cherepanov D: Gastrointestinal neuroendocrine tumors treated with high dose octreotide-lar: A systematic literature review. World J Gastroenterol 21: 1945-1955, 2015. 
35. Welin SV, Janson ET, Sundin A, Stridsberg M, Lavenius E, Granberg D, Skogseid B, Oberg KE and Eriksson BK: High-dose treatment with a long-acting somatostatin analogue in patients with advanced midgut carcinoid tumours. Eur J Endocrinol 151: 107-112, 2004.

36. Ferolla P, Faggiano A, Grimaldi F, Ferone D, Scarpelli G, Ramundo V, Severino R, Bellucci MC, Camera LM, Lombardi G, et al: Shortened interval of long-acting octreotide administration is effective in patients with well-differentiated neuroendocrine carcinomas in progression on standard doses. J Endocrinol Invest 35: 326-331, 2012

37. Baldelli R, Barnabei A, Rizza L, Isidori AM, Rota F, Di Giacinto P, Paoloni A, Torino F, Corsello SM, Lenzi A and Appetecchia M: Somatostatin analogs therapy in gastroenteropancreatic neuroendocrine tumors: Current aspects and new perspectives. Front Endocrinol (Lausanne) 5: 7, 2014.
38. Yao JC, Shah MH, Ito T, Bohas CL, Wolin EM, Van Cutsem E, Hobday TJ, Okusaka T, Capdevila J, de Vries EG, et al: Everolimus for advanced pancreatic neuroendocrine tumors. N Engl J Med 364: 514-523, 2011.

39. Kouvaraki MA, Ajani JA, Hoff P, Wolff R, Evans DB, Lozano R and Yao JC: Fluorouracil, doxorubicin and streptozocin in the treatment of patients with locally advanced and metastatic pancreatic endocrine carcinomas. J Clin Oncol 22: 4762-4771, 2004.

40. Sun W, Lipsitz S, Catalano P, Mailliard JA and Haller DG; Eastern Cooperative Oncology Group: Phase ii/iii study of doxorubicin with fluorouracil compared with streptozocin with fluorouracil or dacarbazine in the treatment of advanced carcinoid tumors: Eastern cooperative oncology group study e1281. J Clin Oncol 23: 4897-4904, 2005. 